G U E S T E D I T O R I A L

\title{
Special Section on Quality Control by Artificial Vision
}

\author{
Hamed Sari-Sarraf \\ Texas Tech University \\ Department of Electrical and Computer Engineering \\ Lubbock, Texas 79409 \\ E-mail: Hamed.Sari-Sarraf@ttu.edu
}

\section{David Fofi \\ Université de Bourgogne \\ Le2i UMR CNRS 5158 \\ 12 rue de la Fonderie \\ Le Creusot, 71200 France}

Nelson H. C. Yung

University of Hong Kong

Electrical Engineering Department

Pokfulam Road

Hong Kong

In all areas of science and engineering, applied researchers shoulder a tremendous responsibility, in that, through their work, they demonstrate the discipline's relevance to the betterment of humankind's quality and quantity of life. It is well understood that failure to do so successfully will inevitably lead to the demise of the discipline.

Machine or artificial vision is a subdiscipline of computer vision that deals with its application in industrial settings. In machine vision, researchers draw from fundamental advances in a multitude of fields, such as sensors, optics, photometry, image processing, pattern recognition, and specialized hardware implementation to conceive complex systems that address ever so complicated problems in product characterization and quality control. Owing to advances made in each of the aforementioned fields in the past two decades, machine-vision methodologies have enjoyed a great deal of attention in as many years. Indeed, today it is nearly impossible to imagine industrial settings, such as the semiconductor or automotive industries, without the footprint of vision-based inspection systems. Of course, as we all know, many challenges still remain that require fundamental contributions from the community of machine-vision researchers.

The papers in this special section were solicited from authors with accepted papers in the 8th International Conference on Quality Control by Artificial Vision, which was held in Le Creusot, France, in 2007, as well as from the community of machine-vision researchers at large. Each submitted paper was reviewed by a minimum of three experts, to whom we are thankful for their detailed and timely reviews.

The special section begins with three papers that extend across all subdisciplines within machine vision. The first two of these give overviews of multiresolution analysis, specifically wavelets and surface registration techniques, respectively. The third paper discusses a method by which to assess the performance of defect detection algorithms, a topic that resonates with many in our community. The next four papers discuss interesting methodologies that are likely to have implications beyond the application areas within which they are presented. For example, in the fourth paper, Morel et al. present an efficient method for the calibration of catadioptric sensors, which are gaining in popularity in applications such as video surveillance. Finally, the last two papers deal with vision sys- tems that are intended for very specific purposes; that is, monitoring the spreading of fertilizers in agricultural fields in the first paper and measuring the length of multiple cotton fibers in the second.

We realize that many unresolved problems still remain, but as the papers in this special section demonstrate, the machine-vision community continues to make tremendous strides in utilizing advances in basic sciences to address consequential challenges.

To conclude, we would like to extend our thanks to the Editor-in-Chief of the Journal of Electronic Imaging, Jan Allebach, for affording us the opportunity to organize this special section, and to the editorial staff of the journal for the efficiency and competence with which they discharge their duties.

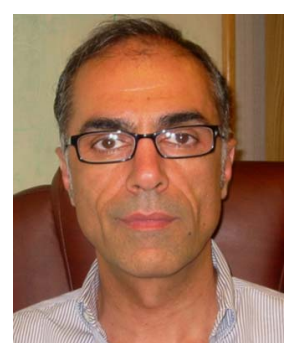

Hamed SariSarraf received his $\mathrm{PhD}$ in electrical engineering from the University of Tennessee, Knoxville, in 1993. After spending a year as a postdoctoral researcher at Oak Ridge National Laboratory (ORNL), he joined the research staff of ORNL's image science and machine 
vision group. In 1999, he accepted a faculty position in the Department of Electrical and Computer Engineering at Texas Tech University where he currently holds the rank of associate professor. He is the director of the Applied Vision Laboratory at Texas Tech University and the Associate Editor of the Journal of Electronic Imaging. His research focuses on the applications of computer vision in industrial monitoring and medical diagnosis.

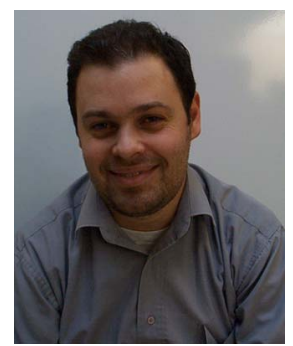

David Fofi studied electric and industrial informatics engineering as an undergraduate at the University of $\mathrm{Pi}$ cardie Jules Verne, Amiens, and received a MSc in image and signal processing from the University of Cergy-Pointoise/ENSEA in 1997. He completed his PhD in the area of coded structured light in 2001 at the Univer- sity of Picardie Jules Verne. He is currently a lecturer at the University of Burgundy and head of the 3-D vision team of the Lab of Electronics, Computer Science and Image Processing. His main research interests are in 3-D vision, projector-camera systems, coded structured light, and catadioptric vision.

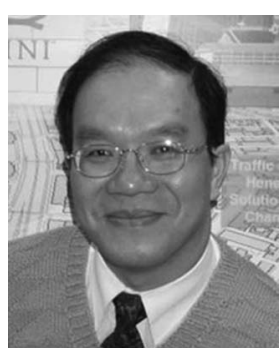

Nelson H. C. Yung received his $B S C$ and $\mathrm{PhD}$ from the University of Newcastle -upon -Tyne, where he was a lecturer 1985 to 1990. From 1990 to 1993 , he worked as senior research scientist in the Department of Defence, Australia. He joined the University of Hong Kong in late 1993 as associate professor. $\mathrm{He}$ is the founding director of the Laboratory for Intelligent Transportation Systems Research at HKU, and also deputy director of HKU's Institute of
Transport Studies. Dr. Yung has coauthored five books and book chapters and has published more than 100 journal and conference papers in the areas of digital image processing, parallel algorithms, visual traffic surveillance, autonomous vehicle navigation, and learning algorithms. He serves as reviewer for the IEEE Transactions on SMC, CASVT, Vehicular Technology, and Signal Processing; Optical Engineering; the Journal of Electronic Imaging; HKIE Proceedings; Microprocessors and Microsystems; and Robotics and Autonomous Systems. He was a member of the advisory panel of the ITS Strategy Review, Transport Department, HKSAR; regional secretary of the IEEE Asia-Pacific region; council member of ITSHK; and chair of the Computer Division of the International Institute for Critical Infrastructures. He is a chartered electrical engineer, member of the HKIE and IEE, and senior member of IEEE. His biography has been published in Who's Who in the World (Marquis, USA). 